\title{
Meanings in motion and faces: Developmental associations between the processing of intention from geometrical animations and gaze detection accuracy
}

\author{
RUTH CAMPBELL, ${ }^{a}$ KATE LAWRENCE, ${ }^{b}$ WILLIAM MANDY, ${ }^{b}$ \\ CHEYTNA MITRA,${ }^{a}$ LALITHA JEYAKUMA,${ }^{a}$ AND DAVID SKUSE ${ }^{b}$ \\ ${ }^{a}$ University College London; and ${ }^{b}$ Institute of Child Health, London
}

\begin{abstract}
Aspects of face processing, on the one hand, and theory of mind (ToM) tasks, on the other hand, show specific impairment in autism. We aimed to discover whether a correlation between tasks tapping these abilities was evident in typically developing children at two developmental stages. One hundred fifty-four normal children (6-8 years and 16-18 years) and 13 high-IQ autistic children (11-17 years) were tested on a range of face-processing and IQ tasks, and a ToM test based on the attribution of intentional movement to abstract shapes in a cartoon. By midchildhood, the ability accurately and spontaneously to infer the locus of attention of a face with direct or averted gaze was specifically associated with the ability to describe geometrical animations using mental state terms. Other face-processing and animation descriptions failed to show the association. Autistic adolescents were impaired at both gaze processing and ToM descriptions, using these tests. Mentalizing and gaze perception accuracy are associated in typically developing children and adolescents. The findings are congruent with the possibility that common neural circuitry underlies, at least in part, processing implicated in these tasks. They are also congruent with the possibility that autism may lie at one end of a developmental continuum with respect to these skills, and to the factor(s) underpinning them.
\end{abstract}

Although many studies have explored differences between people with autism and normally developing controls with respect to sociocognitive processing, the exploration of individual differences in social cognition within normally developing groups is less studied. In this report we were concerned to assess the presence, in midchildhood and again in adolescence, of associations between the ability to interpret information from another person's face (their direction of gaze, their emotional

The authors gratefully acknowledge the support of the National Alliance for Autism Research in funding this work.

Address correspondence and reprint requests to: Ruth Campbell, Department of Human Communication Science, University College London, London UK WC1 N1PF, UK; E-mail: r.campbell@ucl.ac.uk. expression, whether that person has a familiar face) and theory of mind (ToM) skills, using a nonfacial task. We first aimed to test predictions, derived from cognitive neuroscientific literature, of a correlation between specific face-processing skills, especially accuracy in gaze detection, and mentalizing abilities. To explore the validity of our tests in relation to hypotheses derived from autism, in the second phase of the investigation we investigated whether a sample of high functioning autistic adolescents showed specific impairments on these tasks, compared with matched controls.

\section{Gaze Processing and Face Processing in Children and in Autism}

From birth, infants prefer faces with eyes that are open (Batki, Baron-Cohen, Wheelwright, 
Connellan, \& Ahluwalia, 2000) and can discriminate between direct and averted gaze as early as 2-5 days after birth (Farroni, Csibra, Simion, \& Johnson, 2002). Accurate processing of direction of gaze is a requirement for the development of joint attention in infancy and a host of developing behaviors (Moore \& Durnham, 1994). During infancy, autism is often associated with a failure to pay attention to people's faces, and at 1 year of age a failure to make eye contact is the single best discriminator of children who later develop autism (Osterling \& Dawson, 1994). In adulthood, whereas most people inspect the eye region of the face repeatedly and intensively when looking at pictures of people (Yarbus, 1961), individuals with autism do not seem to inspect the eye region any more than other face parts, especially if scanning moving pictures of people (Klin, Jones, Schultz, Volkmar, \& Cohen, 2002). Further, whereas typically developing children have the ability to detect and discriminate direct gaze, children with autism can show impairments in this task (Senju, Yaguchi, Tojo, \& Hasegawa, 2003). However, gaze processing is not the only face-processing task that is disturbed in autism. Many studies confirm that the recognition of identity (e.g., Boucher \& Lewis, 1992; Klin et al., 2002) and of facial expression (e.g., Hobson, 1986) can be specifically impaired. The extent to which processing gaze from faces is associated with processing other facial characteristics is not fully determined at the level of the group or the individual.

\section{Mentalizing}

Mentalizing refers to the ability to interpret actions in terms of the mental states of the agents that instigate and maintain them. Where an interpretation of an event is couched in mental state terms, or where the perceiver makes use of her understanding of the mental state of the agent to describe behavior, we can infer that she has a ToM. At least one plausible hypothesis has been advanced that suggests that mentalizing abilities, which are defective in autism (Baron-Cohen, Leslie, \& Frith, 1985), depend on the ability to process eye contact appropriately from early infancy (Baron-Cohen, 1994, 1995, and see Saxe, Carey, \& Kanwisher, 2004).

\section{A Common Cortical Circuit for Mentalizing and Processing Faces and Where They Are Looking}

The processing of faces for expression and identity activates a predominantly rightlateralized circuit, usually including posterior parts of the superior temporal sulcus (p-STS), ventromedial prefrontal cortex (VMPFC) regions, and temporal pole (see Elgar \& Campbell, 2001). The "gateway" to this system appears to be the middle part of the (right) fusiform gyrus, the fusiform face area (FFA; Kanwisher, McDermott, \& Chun, 1997), which is thought to be highly specialized for the detection of faces and facelike material. The perception of gaze direction appears to make further demands on this circuit, depending on direction of gaze. Inferior frontal regions are activated more readily by faces displaying direct and horizontally averted gaze, than by faces in which gaze is directed downward or when the eyes are closed (Calder et al., 2002). Gaze direction perception also seems to activate $\mathrm{p}$-STS differentially. The p-STS is sensitive to head direction and gaze direction, and to potential movement of eyes relative to head direction (see, e.g., Allison, Puce, \& McCarthy, 2000; Haxby, Hoffman, \& Gobbini, 2002; Kingstone, Tipper, Ristic, \& Ngan, 2004; Puce, Allison, Bentin, Gore, \& McCarthy, 1998; Puce $\&$ Perrett, 2003). Finally, direction of gaze of a pictured face modified FFA activation in a normal population (George, Driver, \& Dolan, 2001).

VMPFC, p-STS, and temporal poles are also implicated in a neural network that is consistently activated, bilaterally, during mentalizing tasks (Frith \& Frith, 2003). However, it is clear that faces are not required, or indeed thought about, for the mentalizing circuit to be activated (Saxe \& Kanwisher, 2003). Thus, two very different types of test of sociocognitive function, the ability to make mental state judgments and the processing of faces, recruit, at least in part, a common neural circuitry. 


\section{Mentalizing animations}

One task that has been used to test mentalizing asks respondents to describe the actions of animated geometrical agents. In the experimental condition, movement patterns of the shapes in relation to each other tend to elicit mental state descriptions ("coaxing," "bullying," "sharing," "a love triangle"). In the control condition the relative movement of the forms elicit interpretations such as "dancing" (Castelli, Happé, Frith, \& Frith, 2000), "bumper car activity" (Schultz et al., 2003), or purely mechanical depictions such as "billiards" (Martin \& Weisberg, 2003). Differential activation (ToM/ social vs. goal directed/mechanical) activated inferior frontal and p-STS regions and the (right) temporal pole in both the Schultz et al. (2003) and Castelli et al. (2000) experiments. Martin and Weisberg (2003), contrasting "social" with "mechanical" animations, also found differential activation in the p-STS and VMPFC.

All three experiments also report differential activity to social movement of abstract shapes in inferior temporooccipital regions, including the fusiform gyrus. This finding suggests that although the middle part of the fusiform gyrus may be specialized for faces, it, and neighboring areas, can be recruited to other sociocognitive visual tasks.

Thus, despite their very different sensorymotor requirements, the viewing of animations that elicit mental state descriptions, and the processing of faces and their gaze, may share some common neural circuitry. One function that may be common to both is related to the detection and interpretation of agency. Certain geometrical animations elicit such interpretations, but the processing of faces also demands the cognitive evaluation of agents whose intentions may be gleaned both from their identity and from their facial movements. How someone looks at a viewer is invested with intentional weight in terms of direction of gaze, pose, and expression (Baron-Cohen, Wheelwright, Hill, Raste, \& Plumb, 2001).

\section{Activation of the Social Brain in Autism}

Several studies have now shown that, in people with autism, activation of the FFA is di- minished or abnormal when they are viewing faces (see Schultz et al., 2000) There are also several studies in which the brains of autistic people were scanned while thinking about the mental state of others (e.g., Baron-Cohen et al., 1999; Castelli, Frith, Happé, \& Frith, 2002; Happé et al., 1996; Saxe \& Kanwisher, 2003). Despite a variety of stimuli and designs, these studies concurred that regions of the social brain associated with mentalizing were less active in the autistic individuals compared with controls, but sensory processing regions were activated normally (see Frith, 2003). Interestingly, Castelli et al. (2002) found that "basal temporal areas" (which presumably included FFA) showed hypoactivation in people with autism compared with controls when they were shown abstract animations that, in typical individuals, elicited ToM interpretations. They reported significantly reduced functional connectivity between extrastriate visual processing regions and areas involved in higher cortical processing of faces such as p-STS.

\section{Predictions}

The notion that a common neural network can be engaged by both mentalizing and face processing suggests that individual differences in these skills may be associated in normally developing people. Further, insofar as both skills appear to be impaired in people with autism, we expect to find specific failure on these tasks in people with autism. Such an outcome would be consistent with the proposal of a continuum of deficit between autism and normal neurocognitive development (Constantino \& Todd, 2003). By contrast, the idea that distinctive neurobiological abnormalities in autism, which independently influence the development of ToM and face-processing abilities (Akshoomoff, Pierce, \& Courchesne, 2002), is neutral with respect to predictions concerning the association or otherwise of these skills in normally developing populations. These may occur, but positive correlational findings fit the "continuum" notion more readily.

In designing this study it was important to select tasks that would provide specific controls for other factors, in particular, tasks that would enable us to focus on the specificity of 
any predicted associations. We chose a task previously used by Castelli and colleagues (Abell, Happé, \& Frith, 2000; Castelli et al., 2000, 2002), which requires descriptions of geometrical animations and has two conditions, only one of which elicits mental state descriptions. We also used three faceprocessing tasks. They included a gaze detection task, a face recognition task, and a task of expression identification. The neurocognitive findings in relation to face processing, as suggested above, are equivocal about the specificity of face-processing task in relation to intention processing: most face-processing tasks engage the "social brain circuit," although differential activation as a function of direction of gaze of the face has also been observed. Functionally, displays of facial emotion have been interpreted as intentional displays (Fridlund, 1991).

The first two experiments concern a group of 90 children, age 7 years (Experiment 1), and a group of more than 60 adolescents, ages 15-17 years (Experiment 2). These large groups permitted regression analysis to be performed on the animation description tasks. Two types of animation display, only one of which reliably elicited mental state descriptions, were shown. The dependent variable was based on individual measures of descriptions of each of these events. These included appropriateness (judged suitability of the description, based the predictions of the test's designers) and intentionality (number of mental state terms). The length (how many words) of each description was also recorded. The predictor variables included IQ and the face tasks. In the third experiment a group of high-functioning adolescents with autism was given all the tasks, and their performance compared with closely matched students from the normal population. We predicted that in normally developing children and adolescents, mentalizing descriptions of animations would be associated with face-processing skills. Which specific face processes would be associated with intention descriptions in normal populations was not predicted at this point. Finally, in line with previously reported studies, we predicted that in youngsters with autism compared with the normal population there may be deficits in face processing, especially gaze, and in ToM descriptions of action.

\section{Experiment 1}

Children aged around 7 years were tested on a number of face-processing skills and on descriptions of geometrical animations. Studies that have examined children's descriptions of animations (see Abell et al., 2000; Bowler \& Thommen, 2000) suggest that there is an increase in the use of mental state terms in describing animations from midchildhood and an increase in appropriateness of use of those terms with age. No study to date has examined the relationship of performance on these tasks with other sociocognitive abilities.

\section{Methods and participants}

Participants. Ninety children aged between 6 and 8 years were recruited from London schools, as part of a larger study of the development of sociocognitive skills. All participants gave their informed consent, via parental statement, prior to their inclusion in the study, and schools were reimbursed for permission to agree testing. The children were screened for inclusion and were excluded if their verbal IQ was below 70. Forty-three students were male, the rest were female. The study was approved by the Institute of Child Health and Great Ormond Street joint ethics committee and was performed in accordance with the ethical standards laid down in the 1964 Declaration of Helsinki.

\section{Materials and test variables.}

Attributing mental states to animated shapes. These animations have previously been used in research on children (Abell et al., 2000) and adults, including adults with autism (Castelli et al., 2000, 2002). Eight silent cartoons, devised by Frith and Happé, each featuring a large red triangle and a smaller blue triangle, were shown on a computer screen. Each animation lasted approximately $40 \mathrm{~s}$. Two conditions were used, with four animations in each condition. In both, the actions of the two fig- 
ures (triangles) in the cartoon appear to be related. In one type of animation (goal directed), the actions of one object showed a simple dependency on those of the other, so each triangle responded to one action pattern with a similar one. For example, the movement of the blue triangle elicited a similar movement pattern in the red one. This type of action tended to elicit goal-directed action terms (fighting, dancing). A second type of action pattern (ToM) decoupled the movements of one object from those of the other, generating a more complex pattern of actions. These scenarios were designed by their authors to illustrate possible intentional relationships between the triangular agents. For instance, a script for the cartoon designed to evoke the term "coaxing" reads: "Mother wants her timid child to get out of the house and play in the garden. The child does not really want to go and resists at first. Eventually mother coaxes him to go out. He explores a bit and finds he likes it. Finally, both dance outside happily. The feelings evoked by this sequence include impatient, anxious, pushy, whining, surprised and happy" (U. Frith, personal communication). These script scenarios, devised by the authors of the test, were the basis for judging the appropriateness of the participant's response.

Although action contingencies differed between the cartoon types, basic visual characteristics in terms of shape and number of agents, general dynamic characteristics, and orientation changes were similar. Participants were read the task instructions as reported by Castelli et al. (2000, 2002), which required the participants to describe what they thought the triangles were doing. Each animation was presented in random order. Participants' spoken responses were recorded, transcribed, and scored. Following Castelli et al., responses to each animation were scored on three dimensions: intentionality, appropriateness, and length. The intentionality score measured degree of intentional attribution (range $=0-5$ ), with absence of intentional language at one extreme and elaborate use at the other. The appropriateness score had a range of $0-2$, with incorrect according to the test authors' scripts at one extreme and highly appropriate at the other. ${ }^{1}$ The third variable was length of description (range $=0-4$, ranging from no response to four or more clauses). Each participant's response to every animation was scored independently by two trained raters. Interrater agreement for intentionality scores was good for both ToM (intraclass correlation $[\mathrm{ICC}]=0.91)$ and goal-directed $(\mathrm{ICC}=0.91)$ animations. Similarly, there was a high level of agreement between raters on appropriateness scores (ToM, ICC $=0.86$; goal directed, ICC $=0.89) .{ }^{2}$ Where discrepancy did occur, a consensus was reached through discussion between raters.

Wechsler Abbreviated Scale of Intelligence (WASI). The Vocabulary and Matrix Reasoning subtests of the WASI were administered according to standardized procedures (Wechsler, 1999). The $T$ scores from these were computed and used to estimate verbal and performance IQ, respectively. An overall IQ score was also derived.

Face-processing tests. The Warrington and Gaze tasks were mounted on a laptop PC using in-house software to display stimuli and collect responses. The expression task was presented using laminated individual portraits as photoimages (gray scale).

1. Warrington test of face recognition: this normed test of face recognition (Warrington, 1984) presents 50 halftone photographs of male faces, as head and shoulders portraits, for later recognition. The images were shown one at a time for the decision "is he nice or nasty?" The experimenter presented a new image as each response was made. When all had been shown, the images were presented again one at a time, but now paired with a new, similar facial image. The respondent indicated which face in each pair had been seen in the earlier presentation.

1. The appropriateness range was reduced from 3 (Castelli et al., 2002) to 2 on the advice of that author.

2. These ICCs were derived using data from a randomly sampled subset $(n=79)$ of the sample from Experiments 1 and 2 . 
Table 1. Experiment 1 descriptive statistics

\begin{tabular}{lcccccrrr}
\hline \hline & \multicolumn{3}{c}{ Males $(n=43)$} & & \multicolumn{2}{c}{ Females $(n=47)$} \\
\cline { 2 - 3 } & Range & Mean & $S D$ & & Range & Mean & $S D$ \\
\hline Age (years) & $6.1-7.9$ & 6.9 & 0.6 & & $6.1-8$ & 7.0 & 0.6 \\
Verbal IQ & $73-116$ & 94.7 & 10.9 & & $72-134$ & 97.3 & 14.3 \\
Full IQ & $77-139$ & 98.0 & 12.2 & & $77-139$ & 100.1 & 14.4 \\
Warrington face recognition & $18-45$ & 32.7 & 5.8 & & $23-49$ & 33.8 & 5.5 \\
Eye gaze accuracy (\%) & $23.3-70$ & 41.3 & 12.7 & & $10-60$ & 39.2 & 13.2 \\
Ekman expression accuracy & $24-51$ & 36.8 & 7.1 & & $27-58$ & 40.4 & 7.0 \\
Goal directed & & & & & & \\
$\quad$ Intentionality & $4-12$ & 9.0 & 1.7 & & $6-14$ & 10.1 & 2.2 \\
$\quad$ Appropriateness & $2-7$ & 4.7 & 1.3 & & $2-8$ & 5.3 & 1.5 \\
$\quad$ Length & $4-16$ & 13.7 & 3.0 & & $6-16$ & 13.4 & 3.0 \\
Theory of mind & & & & & & & \\
$\quad$ Intentionality & $4-15$ & 9.9 & 2.4 & & $6-17$ & 10.5 & 2.6 \\
$\quad$ Appropriateness & $0-5$ & 2.8 & 1.3 & & $0-5$ & 2.8 & 1.3 \\
$\quad$ Length & $2-16$ & 14.6 & 2.9 & & $4-16$ & 14.1 & 2.8 \\
\hline \hline
\end{tabular}

2. Facial expression categorization: the Ekman-Friesen Test of Affect Recognition (Ekman \& Friesen, 1976) was administered. Subjects were shown 60 halftone photographs of emotionally expressive portraits. Ten individual sitters each posed six basic emotions: happiness, surprise, fear, sadness, disgust, and anger. The six emotion labels were presented below each portrait, and participants were asked to decide which emotion was portrayed. The dependent variable was total accuracy of response.

3. Egocentric gaze task: head and neck photographs of a male and female sitter were digitally captured. The sitter inspected four locations in the plane of line-of-sight of the camera. These were straight ahead, left $2^{\circ}$, left $4^{\circ}$, and left $8^{\circ}$. Mirror images of the stimuli were created, producing images of the opposite gaze direction. The test comprised 9 direct gaze, $112^{\circ}$ gaze, $54^{\circ}$ gaze, and $58^{\circ}$ gaze photographs. Images were presented on a computer monitor in a random order. Subjects were required to indicate whether they thought the person in the photograph was looking directly at them, to their left or to their right. The dependent variable was the accuracy of this response across all gaze conditions.

\section{Results}

Descriptive statistics for all the relevant tasks are shown in Table 1. Two tests showed an effect of gender: At 6-8 years of age boys were poorer than girls at discriminating facial expressions in an independent samples test, $t(88)=2.44, p<.02$, and had lower goaldirected intentionality scores, $t(88)=2.51$, $p<.02$. However, these main effects of gender failed to affect subsequent analyses and will not be discussed further here. ${ }^{3}$

Do the two animation conditions generate different scores? Paired-sample $t$ tests for intention score and appropriateness showed significant differences as a function of animation type. There were more intention descriptions for ToM than for goal-directed animations, $t(89)=-2.52, p<.02$. Their appropriateness was lower, $t(89)=12.77$, $p<.001$. Nonparametric $t$ tests (Wilcoxon) on the length scores (which were not normally distributed) showed that ToM descriptive clause length was longer, $z(92)=-4.13$, $p<.001$.

3. Lawrence et al. (personal communication) explore the development of these face-processing tasks with age and gender across childhood and adolescence. 
Correlations between tasks and participant characteristics. Correlations of the participant variables, IQ and age, with each of the task scores were inspected. Age (over this small range) showed no relationship to performance, except for one face task: gaze accuracy correlated highly with age, $r(90)=0.4, p<.001$. All the animation variables, except for ToM length, showed a significant relationship with full IQ. Of the face-processing tasks, only the categorization of facial expression correlated with IQ, $r(90)=0.33, p<.001$.

\section{Prediction of ToM scores by face-processing} variables. As a first step, and for simplification, a combined score was derived for goal-directed animation scores and for ToM animation scores. This was calculated simply by the addition of the intention and appropriateness scores for each animation type. ${ }^{4}$ In this way just two composite variables were calculated: goal-directed (total) and ToM (total) scores.

Following recommended practice for regression analysis (Tabachnick \& Fidell, 2001), the data were first examined for normality and for outliers. Each variable was examined in turn. Kurtosis and skew were within acceptable limits. There was one outlier on one variable: a score of just 10 for gaze accuracy was more than $2 S D$ from the mean. The reported analyses exclude this individual. It should be noted that there were no material differences in the outcome when that data point was included in analysis: that is, the findings reported here were robust with respect to this outlier (see Figure 1). Simultaneous regression is the most conservative form of linear regression analysis, calculating the variance accounted for by each predictor separately. Thus, simple simultaneous regression analysis allowed the possible contribution of IQ

4. Length scores were not incorporated, because previous studies (Abell et al., 2000; Castelli et al., 2002) suggested that they were not particularly salient or reliable measures of mentalizing. Furthermore, the length variable was not normally distributed, showing kurtosis and skew values greater than 3 . This makes interpretation of any parametric analyses that include length scores problematic. They are used only rarely in the present study for this reason. and of the face task to the variance on each of the animation scores to be assessed. Presuppositions for reliable regression analysis are met by the data. For a total number of around 90 , up to six predictor variables may be entered safely into each analysis (Tabachnick \& Fidell, 2001).

Two analyses (Statistical Package for the Social Sciences linear regression) were performed. The dependent variable in each was the animation score: either goal directed (total) or ToM (total), and the predictor variables for each analysis were age, IQ, face recognition, facial expression accuracy, and gaze accuracy. Age was entered as a predictor variable because of its relationship with gaze accuracy. IQ was entered because correlations suggested a relationship between ToM intention scores and IQ, as well as between gaze and IQ, and face recognition and IQ.

When goal-directed scores were the dependent variable, the regression model failed to predict any of the variance. When the dependent variable was ToM total score, the set of predictors predicted a significant proportion of the variance. The $R^{2}$ change was 0.293 ; analysis of variance (ANOVA), $F(5,88)=$ $6.88, p<.001$. However, this does not tell us which of the variables (appropriately weighted) were the best predictors of ToM scores. Inspection of values showed that only two factors contributed significantly to the model. These were IQ $(\beta=0.32, t=3.2, p=.002)$ and gaze accuracy $(\beta=0.36, t=3.37, p=$ .001). Were their contributions to the model independent? Partial correlations showed that the relationship between gaze and ToM scores remained significant after IQ was partialed out, $r(86)=0.37, p<.001$, and was the only face-processing task to do so. IQ and gaze appear to contribute separately to variance in ToM animation descriptions.

Figure 1 shows the scattergram relating ToM scores to gaze accuracy (including the outlier gaze score).

Variance in the different face tasks. The face tasks differed in sensitivity; the gaze task showed the greatest variance (i.e., standard deviation), the expression task less and the face recognition task least (Table 1). Were 


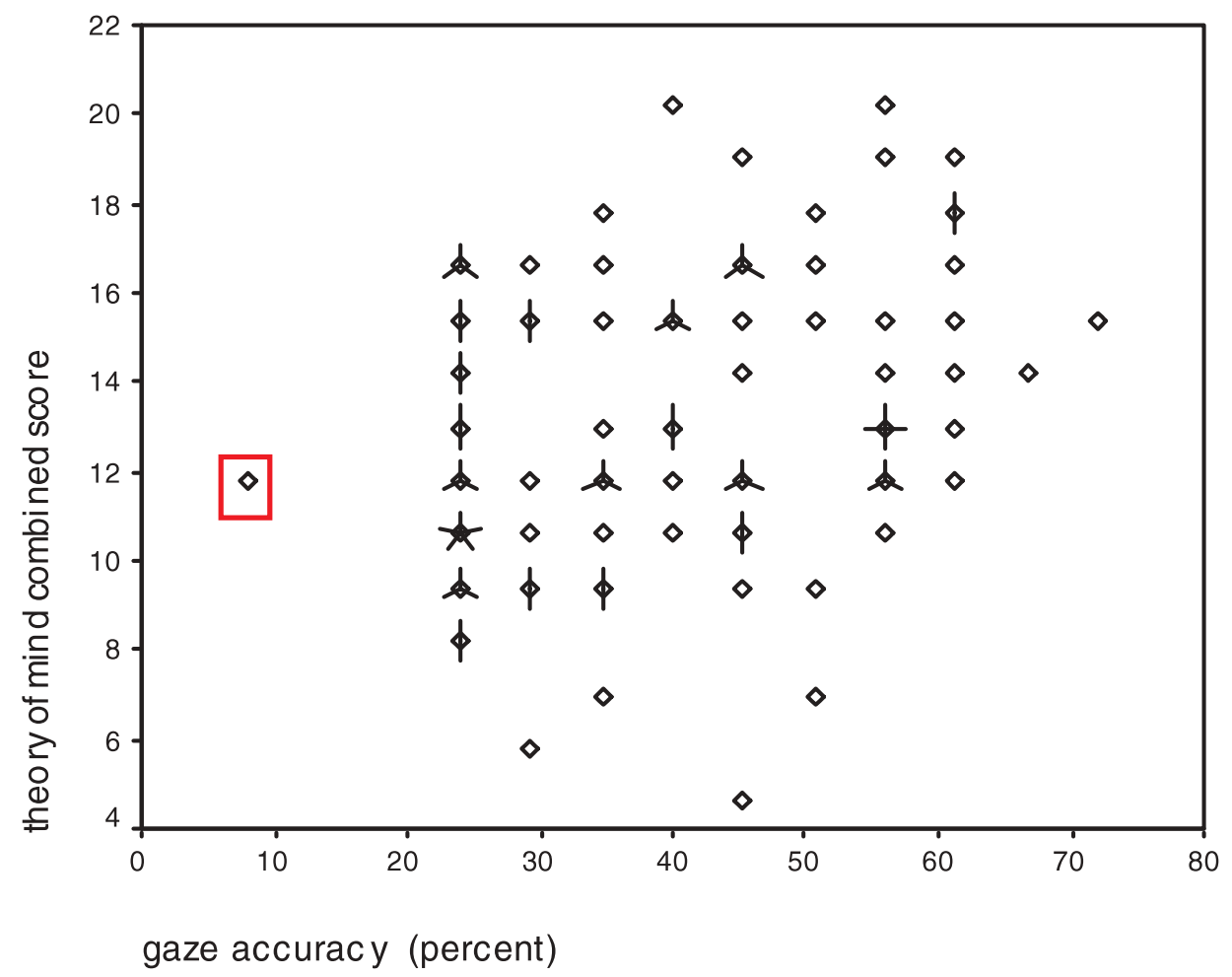

Figure 1. Experiment 1 correlations of ToM scores and gaze accuracy. Outliers excluded from regression analysis are outlined in red. The number of "sunflower petals" indicates the number of cases represented at that data point.

these differences in sensitivity responsible for the specificity of the relationship between gaze and ToM scores? To test this, a sample of participants was selected so that variance in the gaze and the expression tasks was more closely matched. Only those participants with gaze scores below $52 \%$ and above $30 \%$ were included in this sample. In these 45 participants, the mean for the gaze task was $39.36 \%$ $(S D=6.72)$, and that for the expression task was $40.04 \%(S D=6.79)$. That is, variance for the expression task was slightly higher for the expression than for the gaze task in this sample. Correlations for ToM, goal-directed, expression, and gaze scores were calculated, with age and IQ partialed out. Once again, the correlation between gaze and ToM scores was significant $(r=0.34, d f=41, p=.025)$ and was the only significant relationship between face and animation scores. Even when variance in gaze task sensitivity was reduced by eliminating participants with more extreme gaze scores, the relationship with ToM was evident.

Differential sensitivity of these face tasks was unlikely to account for the failure to obtain a relationship between facial expression scores and the animation measures. It is possible that the failure to find a relationship between the animation measures and facerecognition scores reflected the relatively reduced sensitivity of that task. However, face recognition correlated significantly with scores for expression and for gaze, suggesting that the recognition task is sufficiently sensitive to track any relationships that are present.

\section{Discussion}

Normal children in middle childhood reported more "intention-related" ToM than goaldirected descriptions for animations, like adults (Castelli et al., 2000, 2002). Their ToM descriptions were less appropriate, that is, less 
likely to be in accordance with adult norms, than the appropriateness of their goal-directed descriptions. This general pattern is concordant with reported findings for normally developing 8-year-olds, using a somewhat different scoring procedure (Abell et al., 2000).

$I Q$, age, and task performance. IQ had a pervasive effect on the descriptions of animations, and was implicated in some aspects of face processing. This is perhaps less surprising than the finding that age, which ranged around 16 months, showed a very high correlation with accuracy in gaze. This suggests that ability to perform the gaze task emerges quite rapidly around the age of 7 years in normal children.

Intention reading is indexed by specific faceprocessing tasks. Animation descriptions were associated differentially with face processing, and only the animation condition (ToM animations) that elicited mentalizing descriptions showed a relationship with face processing. This relationship persisted when, by further selection of participants, the variance (standard deviation) was matched across the gaze and the expression tasks. No face-reading tasks predicted descriptions for the goal-directed descriptions. Furthermore, only one faceprocessing task, that of gaze accuracy, predicted individual variance of the ToM scores. IQ, although also a powerful predictor of ToM descriptions, was probably not the driver of this effect, because there was no correlation between IQ and gaze in this population.

Measures of descriptions of simple geometrical animations can generate systematic individual differences in 7-year-olds; differences that are associated with a specific measure of social cognition: sensitivity to direction of gaze. This association has not been reported in other studies, and suggests that around this age, the ability to "mentalize" is associated with a simple task of reading directional accuracy in gaze from faces, despite the very different stimulus and task requirements of the tasks. Moreover, the correlations with age suggest this ability is emerging in midchildhood. Further discussion of this relationship is postponed to the General Discussion section. Experiment 2 ex- amines whether a similar pattern may be found in adolescents: that is, is the association between gaze and ToM descriptions transitory, or a sustained trait in development?

\section{Experiment 2}

Experiment 1 was repeated on 16-year-olds. Here, the question was does the same pattern of association between gaze accuracy and mentalizing descriptions of animations hold in this older age group as in the younger group? This age group has not hitherto been examined on the animations description task, where, to date, only 8 -year-olds and adults have been tested.

\section{Methods and participants}

Participants. Sixty-four students (39 male, 25 female) aged between 16 and 18 years were recruited from secondary schools and colleges in the London area under the same recruitment paradigm as described in Experiment 1.

Tasks. The same tasks were administered as described in Experiment 1.

\section{Results}

Descriptive statistics are shown in Table 2 for the 64 participants. Gender had no effect on any of the scores, which are therefore collapsed across that variable.

Do the two animation conditions generate different scores? Paired-sample $t$ tests for intention score and appropriateness showed significant differences as a function of animation type. There were more intention descriptions for ToM than for goal-directed animations, $t(63)=-6.08, p<.001$, but their appropriateness was relatively lower, $t(63)=4.42$, $p=.001$. Nonparametric $t$ tests (Wilcoxon) on the length scores (which, again, were not normally distributed) showed that the descriptive clause length was longer, $z(64)=-4.47$, $p<.001$, for ToM than for goal-directed descriptions. These patterns reiterate those found for 7-year-olds (Experiment 1). 
Table 2. Experiment 2 descriptive statistics $(N=64)$

\begin{tabular}{|c|c|c|c|}
\hline & Range & Mean & $S D$ \\
\hline Age (years) & $15-17.2$ & 16.1 & 0.5 \\
\hline Verbal IQ & $74-142$ & 105.8 & 16.0 \\
\hline Full IQ & $75-133$ & 103.0 & 12.8 \\
\hline $\begin{array}{l}\text { Warrington face } \\
\text { recognition }\end{array}$ & $29-49$ & 41.9 & 4.3 \\
\hline $\begin{array}{l}\text { Eye gaze } \\
\quad \text { accuracy }(\%)\end{array}$ & $43.3-80$ & 61.3 & 7.3 \\
\hline $\begin{array}{l}\text { Ekman expression } \\
\text { accuracy }\end{array}$ & $35-58$ & 49.0 & 5.7 \\
\hline Goal directed & & & \\
\hline Intentionality & $7-17$ & 12.2 & 2.6 \\
\hline Appropriateness & $3-8$ & 5.6 & 1.4 \\
\hline Length & $8-16$ & 13.5 & 2.4 \\
\hline Theory of mind & & & \\
\hline Intentionality & $8-19$ & 14.3 & 2.2 \\
\hline Appropriateness & $0-8$ & 4.6 & 1.5 \\
\hline Length & $8-16$ & 14.9 & 2.0 \\
\hline
\end{tabular}

Patterns of correlation between variables. Age, over this range of approximately 25 months, failed to correlate with any of the other variables. IQ correlated only with animation description length scores and with no other variables. The three face-processing variables showed significant intercorrelations, with the exception of the correlation between gaze accuracy and expression accuracy, which was nonsignificant, $r(n=64)=.17$, $n s$. Intention scores correlated for goal directed and ToM animations. ToM intention and appropriateness scores also showed significant correlations.

Prediction of animation descriptions by face processing. The same procedure as outlined in Experiment 1 was applied to the data here. We hypothesized that some or all of the face-processing tasks would predict individual variance on the ToM combined scores (appropriateness plus intention). We anticipated that, as in the sample of children tested in middle childhood, a similar set of predictors would fail to predict individual differences in variance for goal direction scores. Because the group size was smaller in this than the previous experiment, a maximum of four pre- dictor variables was used in this analysis. Because neither IQ nor age showed a significant relationship with any of the animation description scores except length, these were unlikely to affect the prediction model and were not considered further.

Before analysis, the data were scrutinized for normality. Both the dependent variables (ToM combined score and goal directed combined score) and all the predictor variables (face-recognition accuracy, expression categorization accuracy, and gaze accuracy) were within appropriate range for kurtosis and skew $(< \pm 1)$. The data were inspected for outliers. One outlier was identified with a score $<2 S D$ below the mean for the gaze task and one with a score $>2 S D$ from the mean for this task (see Figure 2). These data points were dropped from subsequent analyses $(N=62)$.

In simple regression analysis, for ToM scores, the model predicted a significant amount of variance, $R^{2}$ change $=0.185 ; \mathrm{AN}$ OVA, $F(3,59)=4.47, p<.01$. The contribution of each of the predictor variables was examined. The $\beta$ for gaze accuracy was significant $(\beta=0.43, t=3.6, p<.001)$, but the corresponding values for face recognition and expression accuracy were not. Gaze accuracy was the main predictor of ToM animations. The correlation between ToM scores and gaze is shown in the scattergram in Figure 2, which includes the two outliers eliminated from regression analysis.

The corresponding analysis was performed for the goal-directed combined animation score. The face-processing predictors failed to predict variance on this factor. Variance (standard deviations) for the gaze task was higher than that of the other two face tasks in this study, as in Experiment 1. To test whether this was the cause of the significant relationships, two participants with extreme scores on the gaze task were eliminated, making variance lower in the gaze $($ mean $=6.45, S D=4.89)$ than expression tasks (mean $=48.37, S D=$ 6.0). The correlation between ToM and gaze scores was significant for this new sample, and, as in Experiment 1, no other relationships between animation and face tasks reached significance. Correlations between all face tasks were significant. Thus, these findings 


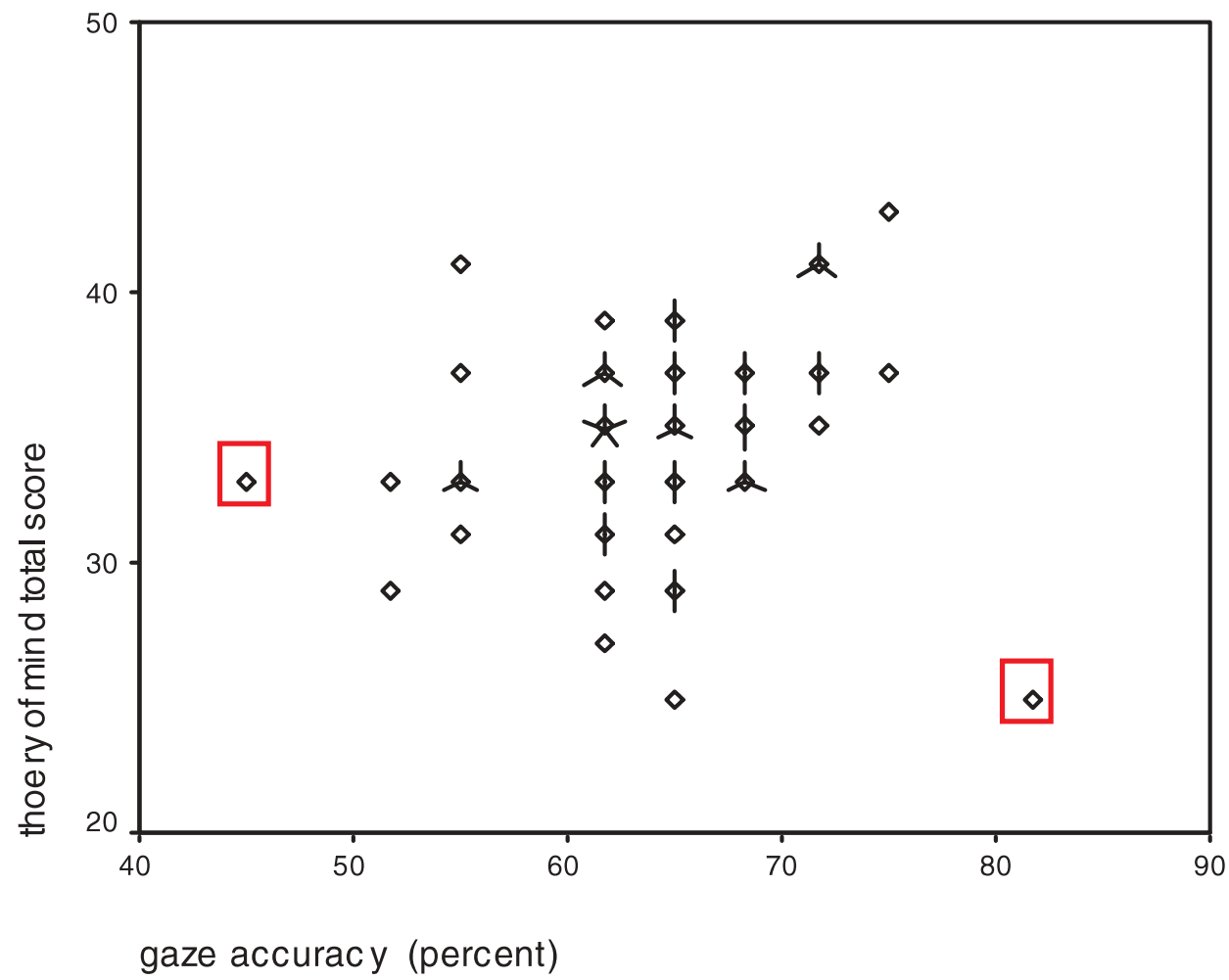

Figure 2. Experiment 2 correlations of ToM scores and gaze accuracy. Outliers excluded from regression analysis are outlined in red. The number of "sunflower petals" indicates the number of cases represented at that data point.

reiterate those of Experiment 1 in an adolescent group, and over an accuracy range very different, for all tasks, than that in younger participants (Experiment 1).

\section{Discussion}

Although they were up to 10 years older than the participants in Experiment 1, and all scores on all tasks were much higher than in younger children, the association between gaze accuracy and ToM animation descriptions is as apparent in this group as in the younger one. This suggests that a single underlying trait or performance factor, which is established by midchildhood, has a pervasive and specific effect on individual sociocognitive differences. Further discussion of this will be deferred to the General Discussion section. A final question posed by these findings concerns the performance of all these tasks by young people with autism, and this is addressed in the next experiment.

\section{Experiment 3}

The previous two experiments in normal populations showed an association between gaze accuracy and ToM descriptions of animations, which is established by around 7 years of age, and is specific to these tasks. This final experiment investigated whether youngsters (aged 11-17) with autism would show deficits specific to these tasks when compared with closely matched controls. In addition to testing faceprocessing and animation descriptions within a single study, we were concerned in this study to examine an age group that has not yet been tested for the animations description task.

Evidence for a specific deficit in animation description in children with autism is inconsistent. Bowler and Thommen (2000) 
explored narrative descriptions of Heider and Simmel's original (1944) geometrical figure action sequences in children with autism and their peers matched by chronological age (10 years) and in children matched by verbal age to the autism group (7 years). They reported no significant difference between the groups in their descriptions of the animations, nor in their use of mental state terms. They did report that the children with autism tended to report animate actions that did not involve contact less often than controls. However, using the same source material, Klin (2000), who employed a social attribution measure to count the incidence of mentalizing terms, did find a deficit in the clinical group. We used different stimuli, which differed in some important characteristics from the Heider and Simmel cartoons. For example, the duration of the events was longer and the scenarios more complex, and the triangles changed shape, as well as moving relative to one another. The animation series used in the present experiments has been investigated by Abell et al. (2000) and Castelli et al. (2000, 2002). Abell et al. tested both goal-directed and ToM animations in children with autism (aged around 13 years) and verbal mental age matched children (aged around 8 years), along with a group of learning disabled children of similar age to those with autism, who also had a mental age of around 8 years. Goal-directed descriptions were more accurate than ToM descriptions in all the younger groups (as replicated in Experiment 1). The number of intentional descriptions did not differ between the three younger groups. However, when the group with autism used mentalizing descriptions they did so less appropriately than the normally developing younger children.

In this experiment we compared a group of high-functioning youngsters (ages 11-17 years) with autism spectrum disorder (ASD) and controls individually matched for age and verbal IQ from the normal population. We predicted that the clinical group may show impaired abilities in their ToM descriptions in the number of intentional descriptions and, following Abell et al. (2000), in their appropriateness. We further hypothesized that some, if not all, faceprocessing skills may be poorer in the group with autism, where differences in face recognition (e.g., Klin et al., 1999) and expression labeling (e.g., Tantam, Monaghan, Nicholson, $\&$ Stirling, 1989) are reliably reported. It should be noted that gaze accuracy, which we found to be a predictor of ToM descriptions in the studies with normal children and adolescents described above, need not be especially compromised in people with autism. Leekam, Baron-Cohen, Perret, Milders, and Brown (1997) found that although children with autism were poor at reading direction of gaze for intention (reading the mind in the eyes; BaronCohen et al., 2001), they were within normal range in identifying where a pictured face was looking in a line-of-sight accuracy task. The present task simply asks participants "where is the face looking," not "what is this person thinking about." However, the engaged gaze accuracy task used in the present study may be more sensitive to autism. In particular, we required the viewer to indicate whether or not gaze was engaged by the pictured face ("Is she looking at you?") rather than "where is she looking" (gaze averted downward and away from camera), which was how line-ofsight was tested by Leekam et al. It should also be borne in mind that several studies find weak or absent evidence that face processing is specifically compromised in children with autism, where more widespread perceptual impairments may be associated with faceprocessing difficulty (Davies, Bishop, Manstead, \& Tantam, 1994).

\section{Method and participants}

Participants. Thirteen adolescents with ASD were recruited from the Social and Communication Disorders Clinic at Great Ormond Street Hospital, London. To meet selection criteria, participants had to be aged between 11 and 17 years, and have a verbal IQ greater than 70 . Diagnosis was established by experienced clinicians, using the The Developmental, Dimensional and Diagnostic Interview, a computerized interview that has been shown to possess good reliability and validity (Skuse et al., 2004). According to this measure, 10 participants met the International Classification of Disease - 10th Edition criteria for child- 
Table 3. Experiment 3 group comparisons

\begin{tabular}{cccccccc}
\hline \hline & & & & & Face & & Goal \\
Group & Age & VIQ & Directed & ToM & $\begin{array}{c}\text { Recog. } \\
(\%)\end{array}$ & $\begin{array}{c}\text { Expression } \\
(\%)\end{array}$ & $\begin{array}{c}\text { Accuracy } \\
(\%)\end{array}$ \\
\hline Control & & & & & & & \\
Mean & 13.3254 & 95.9231 & 17.1538 & 16.8462 & 84.0000 & 80.6944 & 60.2758 \\
$N$ & 13 & 13 & 13 & 13 & 13 & 12 & 12 \\
$S D$ & 2.07529 & 16.40864 & 2.73393 & 2.88231 & 6.97615 & 10.64720 & 7.97204 \\
ASD & & & & & & & \\
Mean & 13.1552 & 96.0769 & 16.6154 & 14.3846 & 74.0000 & 71.3889 & 44.3330 \\
$N$ & 13 & 13 & 13 & 13 & 13 & 12 & 10 \\
$S D$ & 1.75312 & 17.85806 & 2.75495 & 4.19401 & 10.26320 & 10.22063 & 13.15072 \\
\hline \hline
\end{tabular}

Note: Four data points in the experimental tasks were lost prior to analysis; these were due to errors in scoring.

hood autism, with the remaining three being classified as having atypical autism. There were eleven males and two females. Mean age was 13.2 years $(S D=1.8)$ and mean verbal IQ was $96.1(S D=17.9)$.

Control group members were selected individually to match the ASD group in terms of chronological age, verbal IQ, and gender. Mean age was 13.3 years $(S D=2.1)$ and mean verbal IQ was $95.9(S D=16.4)$. Gender composition was identical to that of the ASD group (11 males, 2 females). Controls were recruited from London schools as part of the same study from which participants in Experiments 1 and 2 were drawn.

Materials and test variables. These were the same as those described for Experiment 1, with one exception: ASD participants received a fuller IQ assessment, with all four subtests of the WASI being administered. Thus, the Vocabulary and Similarities subtests were used to estimate verbal IQ, whereas Matrix Reasoning and Block Design subtests were used to give a performance IQ estimate.

\section{Results}

Descriptive statistics are shown in Table 3. All the face-processing tasks, and the combined ToM animations measure, showed significant differences between groups on independent $t$ test. The group effect for gaze accuracy was significant $(t=3.51, d f=24, p<.0001$, one tailed). The effects of face recognition $(t=$ 2.91) and expression categorization $(t=2.18)$ were significant at $p<.01$ (one tailed) and the effect of ToM $(t=1.74)$ was significant at $p<.05$ (one tailed).

Further analyses examined whether specific animation description variables were sensitive to autism and could survive the effects of IQ, which, as Experiment 1 showed, were pervasive in younger participants. These were repeated-measures analyses in which animation type (goal directed or ToM) was one factor and VIQ was the covariate. There were no significant findings for the intention measure, but the appropriateness measure showed a significant effect of group, $F(1,23)=4.91$, which survived covariation for IQ. ToM scores were significantly lower for the group with autism. Relevant means are shown in Table 4.

Correlations. Neither verbal nor nonverbal IQ scores showed a significant correlation with any of the experimental tasks. However, the correlation of verbal IQ and expression classification at $r=.53$ approached significance ( $d f=12, p=.07$, two tailed). The small group sizes precluded separate analyses, with age and IQ partialed out, for each group for each of the experimental tasks.

\section{Discussion}

This study compared high-functioning youngsters with autism with closely matched con- 
Table 4. Experiment 3 group comparisons of means and SDs for the animations description task

\begin{tabular}{cccccccc}
\hline \hline & \multicolumn{3}{c}{ Type 1 } & & & \multicolumn{2}{c}{ Type 2 } \\
\cline { 3 - 4 } Group & Intention & Appropriateness & Length & & Intention & Appropriateness & Length \\
\hline Control & & & & & & & \\
Mean & 11.5385 & 5.6154 & 11.0000 & & 13.0000 & 3.8462 & 13.4615 \\
$N$ & 13 & 13 & 13 & & 13 & 13 & 13 \\
$S D$ & 2.63361 & 1.32530 & 2.82843 & & 1.77951 & 1.67562 & 2.36697 \\
ASD & & & & & & & \\
Mean & 11.6923 & 4.9231 & 12.8462 & & 11.2308 & 3.1538 & 13.2308 \\
$N$ & 13 & 13 & 13 & & 13 & 13 & 13 \\
$S D$ & 2.09701 & 1.03775 & 2.73393 & 2.89119 & 1.72463 & 2.86222 \\
\hline \hline
\end{tabular}

trols with a mean age of 11 years. Despite the close matching of the groups, significant differences emerged that support and complement those of the previous experiments, for they suggest that ToM descriptions and the detection of engaged gaze in a pictured face may be sensitive to a specific developmental factor associated with autism. In this group, although the composite scores revealed group differences in sensitivity to goal-directed and ToM animations, these were influenced by IQ. However, specific ToM appropriateness scores were specifically affected by autism. A similar pattern was reported for adults by Castelli et al. (2002) and, using a different scoring system, by Abell et al. (2000). The finding that direct gaze accuracy was particularly affected by autism concurs with recent reports that, unlike normally developing children, who were better at detecting deviations from engaged than averted gaze in an oddball detection task, children with autism showed no such sensitivity to engaged gaze and deviations from it (Senju et al., 2003), as well as with numerous earlier studies suggesting a specific deficit in managing gaze in normal interaction (e.g., Buitelaar et al., 1991). Facial recognition accuracy was sensitive to autism in this group, as in many other studies (e.g., Klin et al., 1999). Previous studies also find autism affects facial expression categorization (e.g., Teunisse \& de Gelder, 2001), although the simple classification of facial expression may be relatively less vulnerable than the interpretation of expressions in terms of intention and felt emotion (Celani, Battacchi, \& Arcidiacono, 1999; Hobson, 1986, 1991).

Understanding social cues from the eye region of the face has been known to be particularly problematic for people with autism for many years (e.g., Hutt \& Ounsted, 1966; Joseph \& Tanaka, 2002; Langdell, 1978). However, the present task was not explicitly "social," but could be construed as a line-of-sight task, which has been reported to be relatively unaffected in autism (Leekam, Baron-Cohen, Perret, Milders, \& Brown, 1997). One simple interpretation of the gaze deficit in this group is that they were averse to looking at faces, especially when the eyes might sometimes appear to be "looking at you." Post hoc tests established that the significant differences between the groups were accurate for displacements of $4^{\circ}$ and $8^{\circ}$ from center and occurred both for faces seen head on and where the head was laterally averted. These are images where gaze is away from the looker ("over your shoulder"-type gaze), but were interpreted as direct. By contrast, mean accuracy for detecting whether a face looking at the camera really was engaging the viewer's gaze was performed more accurately than the other displacements, and showed no group difference. This pattern suggests a bias, which was more marked in the group with autism, toward categorizing a face as "looking at me" when it was not actually doing so, but showed small lateral angular displacements of gaze.

One theoretical issue relating to the nature of the underlying deficit or deficits in autism 
is addressed by these findings. The autistic cognitive style lacks perceptual coherence, taking relatively more account of local scene details than would occur in normally developing controls (Happé, 1999). It has been suggested that, because faces require configurational processing to be efficiently processed, the deficit in face processing in autism may reflect a lack of central coherence (Frith, 2003). If central coherence were indeed the primary problem in face processing in autism, gaze direction should be spared, rather than specifically impaired. Compared with other face-processing tasks, accuracy in determining direction of gaze is relatively impervious to the face context (Jenkins \& Langton, 2003).

Davies et al. (1994) suggested that apparent impairments in face processing may reflect more general perceptual difficulties in autism. Although their conclusions were with respect to a broader intellectual range of people with autism than was tested here, and no correlation was found between experimental task performance and nonverbal IQ in our sample of ASD people, it is nevertheless interesting to consider whether a perceptual impairment might underlie the poor performance of this group. One way to account for the association between task performance on face processing and animation interpretation is that (some) lowlevel visual deficits in sensitivity to directional motion may be more common in people with autism than in the normally developing cohort (Milne et al., 2002). It is plausible that in the natural environment of the growing infant, sensitivity to eye movements may be critical in establishing accuracy in determining direction of gaze (Farroni, Mansfield, Lai, \& Johnson, 2003). Therefore, any impairment in sensitivity to perceived visual motion may adversely influence the development of more general neural systems concerned with interpreting facial movements. A motion-processing deficit could also influence interpretations of the animations. This paper has not considered how the two animation types differ in their perceptual quality, in particular the demands of different types of contingency detection in space and time needed to make inferences about how two objects in action may relate to each other (Blakemore et al., 2003). Detailed studies of the movement parameters (kinematics) of these animations, and individual sensitivities to them, await investigation.

Other speculations concerning theoretical approaches to "mentalizing" in relation to the patterns of association found in this study are presented at greater length in the following section.

\section{General Discussion}

The picture painted by these studies is consistent. In normal development there is a relationship between accuracy in detection of gaze to the self, and the ability to generate intentional (ToM) descriptions of geometrical animations. This is evident in 7-year-olds and in 16-year-olds, who differ greatly in accuracy of performance on these tasks. These conclusions are specific to one type of animation (animations that generate ToM descriptions in adults) and to one face-processing task (the detection of line-of-sight in a "looking at you" task). This suggests a common factor underlying both tasks. Moreover, these skills were impaired in high-functioning young people with autism, who had been matched closely to controls for verbal IQ and age. Although face recognition and expression classification tasks were also performed poorly by the autism group, performance on the gaze task and ToM descriptions (scored for appropriateness) appeared highly discrepant even within this autistic adolescent group. Taken together, these findings suggest that ability to perform the tasks of gaze detection accuracy and of describing geometrical animations are associated with correlated individual differences. Absolute performance on both tasks varied with age, and with the presence of autistic behavioral traits.

This association between gaze and ToM descriptions of abstract animations is not dependent on any obvious sensory or motor feature of the tasks. The gaze task utilized still images of full faces and required a nonverbal forced-choice response. The animation description score was derived from on-line commentaries given by viewers of a series of clips showing triangles performing contingent actions. The factor common to both tasks must 
relate to higher order aspects of the task and to the interpretation of each of the displays.

Although the face is the source of a great deal of intentional behavior, not all tasks that require the face to be analyzed engage mentalizing processes to the same degree. It might be surprising that the classification of facial expression, which can reflect intentions to act (Frank, 1988) and may have evolved to categorize social displays of dispositions to act (Fridlund, 1991), failed to show any associations with ToM descriptions in children or adolescents. However, Horstmann (2003) found that pictures of facial expressions elicit emotion labels rather than intentional ones in adult respondents. Intentional descriptions dominated only for images of "anger." 5 A further factor adding variability to any relationship of emotion classification with the animations task is the empathic quality of displayed facial emotion. The categorization of facial emotions invokes some of the feelings associated with the displayed emotion, and these are likely to show idiosyncratic differences (Wild, Erb, \& Bartels, 2001).

However, why should gaze to the self- and ToM descriptions of animations be so closely associated, and from such a young age? One skill common to both is sensitivity to the precise direction of attention in other people or agents. The gaze task required the respondent to indicate deviations from engaged gaze, that is, to be sensitive to when the pictured face is or is not "looking at me." Intentional ascriptions of animation also require that the interpreter assess to what, where, and when the viewed agents may be directing their attention, as a prerequisite for determining their intention. The ability to accurately detect the attentional focus of another is a critical component of the development of joint-attention skills in infancy, a skill that is reduced or absent in infants who later are diagnosed with autism (Osterling \& Dawson, 1994).

5. In the adolescent data set, ToM animation scores correlated with "surprise," $r$ (partialed for IQ) $=.28, p<$ .025 , only. The correlation with anger was negative and nonsignificant. There were no correlations of any specific emotions with any of the animation descriptions in children.
An intriguing aspect of sensitivity to gaze in people with autism is that although they are poor at "reading the eyes," they can show essentially normal sensitivity to automatic ("reflexive") social cues. That is, when a visual search target appears in a location that had immediately previously been cued by the direction of gaze of a pictured face, children with autism showed similar sensitivity to controls: both showed improved performance at detecting the target at the cued compared with a noncued location (Chawarska, Klin, \& Volkmar, 2003; Swettenham, Condie, Campbell, Milne, \& Coleman, 2003).

This paradoxical pattern, which is impaired use of line-of-sight as a volitional cue, coupled with relatively normal sensitivity to line-ofsight as a reflexive cue, conforms with another approach to autism, one that stresses autistic difficulties in inhibiting prepotent responses, and in mental flexibility (Hill, 2004; Ozonoff, Pennington, \& Rogers, 1991). The social cue effect, being largely involuntary, may have a basis in relatively low level attentional mechanisms that need not be impaired in autism. A similar argument may be made with respect to the processing of animations. To attribute mental states to the figures in motion it is necessary to focus differentially on movement patterns of individual agents in action: to switch from one agent to the other to interpret the contingencies of action appropriately. This requirement is less pronounced in following goaldirected animations, where the actions of the figures are coordinated in a simpler fashion in space and time. To interpret the goal-directed actions accurately, it is sufficient to describe actions of one of the agents, or of the agents as a linked pair. The contingencies of the two types of animation may require different patterns of attentional deployment: in particular, appropriate ToM attributions require flexible switching of attention between movement patterns of individual actions. The requirement for attentional flexibility is absent from face-processing tasks other than gaze tracking. Those other tasks, as we have seen, show weak or absent relationships with ToM animations, despite their sociocognitive value.

This suggestion can be reduced to the following possibility: a child who can readily 
switch between looking at the face and looking at "where the face is looking," to detect regions of interest within the visual scene, will be likely to develop an advantage in developing intentional understanding, which can also require management of complex patterns of attention across events, over one who is either consistently face fixated or face averse, or a child who is consistently captivated by persistent visual qualities of some or other region of the scene itself.

Although an association between gaze sensitivity and mentalizing has been proposed in relation to the early development of social cognitive skills (Baron-Cohen, 1994, 1995), at this stage it would be premature to speculate on the "hidden links" that may connect demonstrations of joint attention in infancy and early childhood to the coordinated performance of the 6-year-old performing the "look at me" and the "triangles" task. Nor could experiments be done easily to track this development. The argument above sketches out some reasons for this: the behavioral repertoire of the infant and the young child is such that it may be difficult to distinguish volitional from automatic responsiveness to gaze cues (although electrophysiological measurements may be helpful here; see Farroni, Johnson, \& Csibra, 2004). Language development, especially exposure to conversation, is implicated in ToM descriptions, whether tested using verbal or nonverbal descriptions (e.g., Peterson, 2004; see Saxe et al., 2004, for review). However, our argument suggests that sociocognitive development, whether tracked by gaze sensitivity or by intentional projections in descriptions, may be scaffolded upon perceptual abilities, and that these may be manifest relatively early in development as patterns of direction of gaze, especially within a social context. The rate of development of these perceptual abilities may itself be responsive to sociocognitive factors. There are many reasons why gaze behaviors may develop according to different trajectories and at different rates (with knock-on implications for a range of social cognitive tasks). Apart from ASD, these are likely to include any of the following: depressed motivational state of the child, negative emotions within the mother-child dyad, difficulties in managing motivational schedules for the child, and communicative problems, including childhood deafness. One task for the future is to explore the extent to which the association between gaze detection accuracy and mentalizing descriptive skills holds when these factors become paramount.

Our demonstration that the predicted association was found in normally developing 6-year-olds suggests, at the very least, that the causal mechanisms for the association are relatively unaffected by the contingencies of middle and later childhood (schooling, literacy, changing patterns of engagement with the adult world) and will have their roots in events in infancy and early childhood. Individual differences in such sensitivity may have a genetic basis, and may require that an early predisposition to potentially socially important events (including faces and biological movement) is evident early in infancy. These suppositions remain to be tested at the level of individual differences.

The hypotheses that drove this investigation were derived in large part from neuroimaging findings that had indicated a common cortical circuit for the processing of animated movements requiring interpretations of the mental state and the processing of faces. We predicted that intercorrelations would be found between all these abilities in typically developing children, and that these skills may be specifically affected in autism. Not all faceprocessing tasks correlated with intentional descriptions of animations, although performance on the three face tasks tended to be strongly intercorrelated. Face recognition and facial expression classification accuracy were not correlated with ToM abilities in typically developing children or adolescents. That is, the circuits for analyzing faces and for interpreting animations in intentional terms are partly dissociable. This dissociation is intriguing, indicating that within the "social brain" there are distinct developmental pathways that play different roles in extracting social information from faces.

In autistic adolescents, relations between face-processing tasks and ToM abilities await fuller investigation with larger groups, but the finding that gaze perception was not the only 
face-processing task to be impaired in this group suggests the appropriate specialization of relevant neural circuits had not occurred. Failures of developmental specialization for different sociocognitive tasks may be hypothesized to underlie these patterns, which await further investigation at the neuroimaging and behavioral levels.

\section{References}

Abell, F., Happé, F., \& Frith, U. (2000). Do triangles play tricks? Attribution of mental states top animated shapes in normal and abnormal development. Cognitive Development, 15, 1-20.

Akshoomoff, N., Pierce, K., \& Courchesne, E. (2002). The neurobiological basis of autism from a developmental perspective. Development and Psychopathology, 14, 613-634.

Allison, T., Puce, A., \& McCarthy, G. (2000). Social perception from visual cues: Role of the STS region. Trends in Cognitive Science, 4, 267-278.

Batki, A., Baron-Cohen, S., Wheelwright, S., Connellan, J., \& Ahluwalia, J. (2000). Is there an innate gaze module? Evidence from human neonates. Infant Behavior and Development, 23, 223-229.

Baron-Cohen, S. (1994). How to build a baby that reads minds: Cognitive mechanisms in mindreading Current Psychology of Cognition, 13, 513-552.

Baron-Cohen, S. (1995). Mind-blindness: An essay on the psychology of autism. Cambridge, MA: MIT Press.

Baron-Cohen, S., Leslie, A. M., \& Frith, U. (1985). Does the autistic child have theory of mind? Cognition, 21, 37-46.

Baron-Cohen, S., Ring, H. A., Wheelwright, S., Bullmore, E. T., Brammer, M. J., Simmons, A., et al. (1999). Social intelligence in the normal and autistic brain: An fMRI study. European Journal of Neuroscience, 11, 1891-1898.

Baron-Cohen, S., Wheelwright, S., Hill, J., Raste, Y., \& Plumb, I. (2001). The "Reading the Mind in the Eyes" Test revised version: A study with normal adults, and adults with Asperger syndrome or high-functioning autism. Journal of Child Psychology and Psychiatry, 42, 241-251.

Blakemore, S. J., Boyer, P., Pachot-Clouard, M., Meltzoff, A., Segebarth, C., \& Decety, J. (2003). The detection of contingency and animacy from simple animations in the human brain. Cerebral Cortex, 13, 837-844.

Boucher, J., \& Lewis, V. (1992). Unfamiliar face recognition in relatively able autistic children. Journal of Child Psychology and Psychiatry, 33, 843-859.

Bowler, D. M., \& Thommen, E. (2000). Attribution of mechanical and social causality to animated displays by children with autism. Autism, 4, 147-171.

Buitelaar, J. K., van Engeland, H., de Kogel, K. H., de Vries, H., \& van Hooff, J. A. (1991). Differences in the structure of social behavior of autistic children and non-autistic retarded controls. Journal of Child Psychology and Psychiatry, 32, 995-1015.

Calder, A. J., Lawrence, A. D., Keane, J., Scott, S. K., Owen, A. M., Christoffels, I., et al. (2002). Reading the mind from eye gaze. Neuropsychologia, 40, 1129-1138.

Castelli, F., Frith, C., Happé, F., \& Frith, U. (2002). Autism, Asperger syndrome and brain mechanisms for the attribution of mental states to animated shapes. Brain, 125, 1839-1849.

Castelli, F., Happé, F., Frith, U., \& Frith, C. (2000). Movement and mind: A functional imaging study of perception and interpretation of complex intentional movement patterns. Neuroimage, 12, 314-325.

Celani, G., Battacchi, M. W., \& Arcidiacono, L. (1999). The understanding of the emotional meaning of facial expressions in people with autism. Journal of Autism and Developmental Disorders, 29, 57-65.

Chawarska, K., Klin, A., \& Volkmar, F. (2003). Automatic attention cueing through eye movement in 2-year-old children with autism. Child Development, $74,1108-1122$.

Constantino, J. N., \& Todd, R. D. (2003). Autistic traits in the general population: A twin study. Archives of General Psychiatry, 60, 524-530.

Davies, S., Bishop, D., Manstead, A. S., \& Tantam, D. (1994). Face perception in children with autism and Asperger's syndrome. Journal of Child Psychology and Psychiatry, 35, 1033-1057.

Ekman, P., \& Friesen, W. (1976). The pictures of facial affect. Palo Alto, CA: Consulting Psychologists Press.

Elgar, K., \& Campbell, R. (2001). Face recognition disorders in developmental neuroscience. Journal of Child Psychology and Psychiatry, 42, 707-725.

Farroni, T., Csibra, G., Simion, F., \& Johnson, M. H. (2002). Eye contact detection in humans from birth. Proceedings of the National Academy of Science of the United States of America, 99, 9602-9205.

Farroni, T., Johnson, M. H., \& Csibra, G. (2004). Mechanisms of eye gaze perception during infancy. Journal of Cognitive Neuroscience, 16, 1320-1326.

Farroni, T., Mansfield, E. M., Lai, C., \& Johnson, M. H. (2003). Infants perceiving and acting on the eyes: Tests of an evolutionary hypothesis. Journal of Experimental Child Psychology, 85, 199-212.

Frank, R. (1988). Passions within reason: The strategic role of the emotions. New York: W. W. Norton.

Fridlund, A. J. (1991). Evolution and facial action in reflex, social motive, and paralanguage. Biological Psychology, 32, 3-100.

Frith, C. (2003). What do imaging studies tell us about the neural basis of autism? In Autism: Neural basis and treatment possibilities. Novartis Symposium 251 (pp. 149-176). Chichester: Wiley.

Frith, U., \& Frith, C. D. (2003). Development and neurophysiology of mentalizing. Philosophical Transactions of the Royal Society of London B, 358, 459-473.

George, N., Driver, J., \& Dolan, R. J. (2001). Seen gazedirection modulates fusiform activity and its coupling with other brain areas during face processing. Neuroimage, 13, 1102-1112.

Happé, F. (1999). Autism: Cognitive deficit or cognitive style? Trends in Cognitive Science, 3, 216-222. 
Happé, F., Ehlers, S., Fletcher, P., Frith, U., Johansson, M., Gillberg, C., et al. (1996). "Theory of mind" in the brain. Evidence from a PET scan study of Asperger syndrome. Neuroreport, 8, 197-201.

Haxby, J. V., Hoffman, E. A., \& Gobbini, M. I. (2002). Human neural systems for face recognition and social communication. Biological Psychiatry, 51, 59-67.

Heider, F., \& Simmel, M. (1944). An experimental study of apparent behavior. American Journal of Psychology, 57, 243-259.

Hill, E. L. (2004). Executive dysfunction in autism. Trends in Cognitive Science, 8, 26-32.

Hobson, R. P. (1986). The autistic child's appraisal of expressions of emotion: A further study. Journal of Child Psychology and Psychiatry, 27, 671-680.

Hobson, R. P. (1991). Methodological issues for experiments on autistic individual's perception and understanding of emotion. Journal of Child Psychology and Psychiatry, 32, 1135-1158.

Horstmann, G. (2003). What do facial expressions convey: Feeling states, behavioral intentions, or action requests? Emotion, 3, 150-166.

Hutt, C., \& Ounsted, C. (1966). The biological significance of gaze aversion with particular reference to the syndrome of infantile autism. Behavioral Science, 11, 346-356.

Jenkins, J., \& Langton, S. R. (2003). Configural processing in the perception of eye-gaze direction. Perception, 32, 1181-1188.

Joseph, R. M., \& Tanaka, J. (2002). Holistic and partbased face recognition in children with autism. Journal of Child Psychology and Psychiatry, 4, 1-14.

Kanwisher, N., McDermott, J., \& Chun, M. M. (1997). The fusiform face area: A module in human extrastriate cortex specialized for face perception. Journal of Neuroscience, 17, 4302-4431.

Kingstone, A., Tipper, C., Ristic, J., \& Ngan, E. (2004). The eyes have it!: An fMRI investigation. Brain and Cognition, 55, 269-271.

Klin, A. (2000). Attributing social meaning to ambiguous visual stimuli in higher functioning Autism and Asperger syndrome: The social attribution task. Journal of Child Psychology and Psychiatry, 41, 831-846.

Klin, A., Jones, W., Schultz, R, Volkmar, F., \& Cohen, D. (2002). Visual fixation patterns during viewing of naturalistic social situations as predictors of social competence in individuals with autism. Archives of General Psychiatry, 59, 809-816.

Klin, A., Sparrow, S. S., de Bildt, A., Cicchetti, D. V., Cohen, D. J., \& Volkmar, F. R. (1999). A normed study of face recognition in autism and related disorders. Journal of Autism and Developmental Disorder, 6, 499-508.

Langdell, T. (1978). Recognition of faces: An approach to the study of autism. Journal of Child Psychology and Psychiatry, 19, 255-268.

Leekam, S., Baron-Cohen, S., Perret, D., Milders, M., \& Brown, S. (1997). Eye-direction detection: A dissociation between geometric and joint attention skills in autism. British Journal of Developmental Psychology, 15, 77-95.

Martin, A., \& Weisberg, J. (2003). Neural foundations for understanding social and mechanical concepts. Cognitive Neuropsychology, 20, 575-587.

Milne, E., Swettenham, J., Hansen, P., Campbell, R., Jeffries, H., \& Plaisted, K. (2002). High motion coherence thresholds in children with autism. Journal of Child Psychology and Psychiatry, 43, 255-263.
Moore, C., \& Durnham, P. (Eds.). (1994). Joint attention: Its origins and role in development. Hillsdale, $\mathrm{NJ}$ : Erlbaum.

Osterling, J., \& Dawson, G. (1994). Early recognition of children with autism: A study of first birthday home videotapes. Journal of Autism and Developmental Disorder, 24, 247-257.

Ozonoff, S., Pennington, B. F., \& Rogers, S. J. (1991). Executive function deficits in high-functioning autistic individuals: Relationship to theory of mind. Journal of Child Psychology and Psychiatry, 32, 1081-1105.

Peterson, C. C. (2004). Theory-of-mind development in oral deaf children with cochlear implants or conventional hearing aids. Journal of Child Psychology and Psychiatry, 45, 1096-1106.

Puce, A., Allison, T., Bentin, S., Gore, J. C., \& McCarthy, G. (1998). Temporal cortex activation in humans viewing eye and mouth movements. Journal of Neuroscience, 18, 2188-2199.

Puce, A., \& Perrett, D. (2003). Electrophysiology and brain imaging of biological motion. Philosophical Transactions of the Royal Society of London B, 358, 435-445

Saxe, R., Carey, S., \& Kanwisher, N. (2004). Understanding other minds: Linking developmental psychology and functional neuroimaging. Annual Review of Psychology, 55, 87-124.

Saxe, R., \& Kanwisher, N. (2003). People thinking about thinking people. The role of the temporo-parietal junction in "theory of mind." Neuroimage, 19 , 1835-1842.

Schultz, R. T., Gauthier, I., Klin, A., Fulbright, R. K., Anderson, A. W., Volkmar, F., et al. (2000). Abnormal ventral temporal cortical activity during face discrimination among individuals with autism and Asperger syndrome. Archives of General Psychiatry, 57, 331-340.

Schultz, R. T., Grelotti, D. J., Klin, A., Kleinman, J., Van der Gaag, C., Marois, R., et al. (2003). The role of the fusiform face area in social cognition: Implications for the pathobiology of autism. Philosophical Transactions of the Royal Society of London B, 358, 415-427.

Senju, A., Yaguchi, K., Tojo, Y., \& Hasegawa, T. (2003). Eye contact does not facilitate detection in children with autism. Cognition, 89, B43-B51.

Skuse, D., Warrington, R., Bishop, D., Chowdhury, U., Lau, J., Mandy, W., et al. (2004). The Developmental, Dimensional and Diagnostic Interview (3Di): A novel computerized assessment for autism spectrum disorders. Journal of American Academy of Child and Adolescent Psychiatry, 4, 548-558.

Swettenham, J., Condie, S., Campbell, R., Milne, E., \& Coleman, M. (2003). Does the perception of moving eyes trigger reflexive visual orienting in autism? Philosophical Transactions of the Royal Society of London B, 358, 325-334.

Tabachnick, B. G., \& Fidell, L. S. (2001). Using multivariate statistics. Needham, MA: Allyn \& Bacon.

Tantam, D., Monaghan, L., Nicholson, H., \& Stirling, J. (1989). Autistic children's ability to interpret faces: A research note. Journal of Child Psychology and Psychiatry, 30, 623-630.

Teunisse, J. P., \& de Gelder, B. (2001). Impaired categorical perception of facial expressions in high-functioning adolescents with autism. Neuropsychology Develop- 
mental Cognition Section C Child Neuropsychology, $7,1-14$.

Warrington, E. K. (1984). The recognition memory test. London: NFER-Nelson.

Wechsler, D. (1999). Wechsler Abbreviated Scale of Intelligence (WASI). San Antonio, TX: Psychological Corporation.
Wild, B., Erb, M., \& Bartels, M. (2001). Are emotions contagious? Evoked emotions while viewing emotionally expressive faces: Quality, quantity, time course and gender differences. Psychiatry Research, 102, 109-124.

Yarbus, A. L. (1961). Eye movements during the examination of complicated objects. Biofizika, 6, 52-56. 